LPT-ORSAY 03-52

\title{
Symplectic structure for elastic and chiral conducting cosmic string models
}

\author{
B. Carter ${ }^{\sharp}$ and D. A. Steer ${ }^{b}$ \\ ${ }^{\sharp}$ LuTh, Observatoire de Paris-Meudon, 92195 Meudon, France. \\ ${ }^{b}$ Laboratoire de Physique Théorique ${ }^{1}$ \\ Bât. 210, Université Paris XI, 91405 Orsay Cedex, France \\ and \\ Fédération de recherche APC, Université Paris VII, \\ 2 place Jussieu, 75251 Paris Cedex 05, France.
}

July 2003

\begin{abstract}
This article is based on the covariant canonical formalism and corresponding symplectic structure on phase space developed by Witten, Zuckerman and others in the context of field theory. After recalling the basic principles of this procedure, we construct the conserved bilinear symplectic current for generic elastic string models. These models describe current carrying cosmic strings evolving in an arbitrary curved background spacetime. Particular attention is paid to the special case of the chiral string for which the worldsheet current is null. Different formulations of the chiral string action are discussed in detail, and as a result the integrability property of the chiral string is clarified.
\end{abstract}

\footnotetext{
${ }^{1}$ Unité Mixte de Recherche du CNRS (UMR 8627).
} 


\section{Introduction}

Following a preliminary study [1] whose application was restricted to the nonconducting Nambu Goto (NG) strings, in this article we focus on conducting cosmic string models and apply the general principles of covariant canonical variational analysis. As emphasised by Witten, Zuckerman, and others [2, 3, 4, 5, 6, 7, 8] in the context of relativistic field theories, the potential utility of this covariant analysis is as a starting point for covariant quantization. Despite that, our aim is not to quantize. Rather, the culmination of this analysis is the construction of a corresponding symplectic structure which is locally representable as a current. This symplectic current - which should not be confused with the world-sheet current of the conducting string - is defined as an antisymmetric bilinear functional of a pair of independent perturbations, and it is conserved whenever both perturbations are on shell (in the sense of satisfying the relevant dynamical field equations).

The task of extending such analysis from ordinary fields to branes (meaning systems with support confined to a lower dimensional worldsheet) was recently taken up by Cartas-Fuentevilla [9, 10]. The necessary analysis is facilitated by the relatively new development [11, 12, 13] of suitably covariant methods of geometrical analysis, which have already been shown to be far more efficient than the more cumbersome (and error prone) frame dependent methods used in earlier work for treating other problems, such as the divergences arising from self interaction [14, 15, 16].

The preceding article [1] demonstrates the agreement of the canonical approach, as developed by Cartas-Fuentevilla [9, 10, with the result of an earlier and rather different approach [1] to the construction of the symplectic surface current. Both these analyses focused on strings with no internal physical structure on the worldsheet, that is on actions of the simple Dirac-Nambu-Goto type which are proportional to the worldsheet surface measure.

The present work deals with the more general case of elastic string models of the kind [17] appropriate for macroscopic applications, such as the strings used for musical instruments since the time of Pythagorus, and in particular for the macroscopic description of the effect in cosmic strings of mechanisms of the various (fermionic and bosonic superconducting) kinds originally proposed by Witten [18. The earliest work on the consequences of the Witten mechanism emphasised effects due to electromagnetic coupling, but it was recognised by Davis and Shellard [19] that the most important consequence would be the formation of vortons, meaning centrifugally supported equililibrium configurations of loops in which effects of electromagnetic coupling (if present at all) are relatively unimportant. While it is also possible to have non-circular vorton configurations [20, the simplest possibility is that of circular configurations. In this case (provided electromagnetic effects are absent or negligible) both the static equilibrium and full dynamical evolution is particularly amenable to an exact mathematical treatment: this was provided by a recent study [21] whose notation scheme will be followed here.

This paper is set up in the following way. In section 2 we review the generic elastic string model and the limit case of the chiral model, and derive the relevant equations of motion. Different formulations of the chiral string action are discussed in detail in 
section $2 \mathrm{a}$, and as a result we are able to reinterpret more simply the integrability property of the chiral string. The purpose of section 3 is to summarize the basic steps of the canonical analysis of Witten and others, and this is done in the context of a general worldsheet action density. In section 4 we discuss in which reference system the symplectic current will be evaluated; the result for the general elastic string is given in section 5, and for the chiral string in section 6. Finally this paper contains an appendix in which we use Dirac's Hamiltonian method for constrained systems in order to write the chiral string action in a linearised Polyakov-like form.

Since there are many different indices to keep under control in this analysis, we have tried to clarify the presentation by using a colour scheme. Black indices refer to spacetime quantites; green quantities are geometrical, describing the embedding of the brane (in this case a string) in the background spacetime; and blue indices on a vector run over both spacetime and internal indices. We have decided to write all physical quantities (invariant under gauge transformations and rescalings) in red; generalised momenta are written in brown; and quantities in purple are dynamical (generally gauge or normalisation dependent) variables.

\section{Elastic and chiral string models}

The string models we consider are governed by an action integral of the form

$$
\mathcal{I}=\int L\|\gamma\|^{1 / 2} \mathrm{~d}^{2} \sigma
$$

over a supporting worldsheet with internal coordinates $\sigma^{i}(i=0,1)$ and induced metric $\gamma_{i j}=g_{\mu \nu} x_{, i}^{\mu} x_{, j}^{\nu}$, in a background with coordinates $x^{\mu},(\mu=0,1, \ldots d),(d \geq 2)$ and (flat or curved) space-time metric $g_{\mu \nu}$. In the NG case, the scalar Lagrangian action density $L$ is just a constant. For the more general category [21] of elastic string models considered here, $L$ depends on the magnitude of the gradient of a freely variable phase field $\varphi$. More explicitly the equation of state is $L\{w\}$ where

$$
w=\psi^{2} \gamma^{i j} \varphi_{, i} \varphi_{, j}
$$

with $\psi$ a normalisation parameter with fixed value given

$$
\psi^{2}=\kappa_{0}
$$

The constant $\kappa_{0}$ may be fixed according to convenience without loss of generality of the model: the standard convention [21, however, is to choose $\kappa_{0}$ such that the derived quantity $\mathcal{K}$ defined by

$$
\mathcal{K}^{-1}=-2 \frac{\mathrm{d} L}{\mathrm{~d} w}
$$

tends to unity in the null limit $w \rightarrow 0$ (see equation 13 and section 2 a).

For any such elastic string action there is a corresponding chiral string action which is obtained by relaxing the condition that $\psi$ is fixed, and letting it instead be a freely varying auxiliary field. On application of the variation principle, it follows that the 
on shell configurations satisfy the same dynamical equations as in the corresponding elastic model, though there is now the further constraint that $w=0$. It can be verified that this null constraint is consistent with the dynamical equations: if $w=0$ at an initial time, it will automatically be preserved by the evolution of the system.

This chiral string is of interest 22] since in a number of different and cosmologically relevant cases [23, 24, Witten's fermionic zero mode mechanism indeed gives rise to purely left (or purely right) moving modes and hence to a null current. Furthermore it was recently recognized [25, 26, 27] that the chiral string equations of motion are exact integrable in a flat background (see also further comments on this in section 2). This has led to a number of analyses of the cosmological consequences of chiral cosmic strings [26, 28]. The chiral string can also be thought of as a useful approximation for the treatment of nearby solutions of the less tractable generic elastic class.

As in the simple NG case $(\varphi=0)$, the full set of dynamical equations for the generic elastic case is given by the local surface energy momentum conservation equation, which takes the standard form

$$
\bar{\nabla}_{\mu} T^{\mu \nu}=0 .
$$

Here the surface covariant differentiation operator $\bar{\nabla}$ is defined in terms of the fundamental tensor $\eta^{\mu \nu}$ by

$$
\bar{\nabla}_{\mu}=\eta_{\mu}^{\nu} \nabla_{\nu}, \quad \eta^{\mu \nu}=\gamma^{i j} x_{, i}^{\mu} x_{, j}^{\nu},
$$

and the surface stress momentum energy density is defined by

$$
T^{\mu \nu}=2\|\gamma\|^{-1 / 2} \frac{\partial\left(L\|\gamma\|^{1 / 2}\right)}{\partial g_{\mu \nu}} .
$$

The motion of the worldsheet is governed by the orthogonally projected part of (5.5) which takes the form

$$
T^{\mu \nu} K_{\mu \nu}{ }^{\rho}=0
$$

where $K_{\mu \nu}{ }^{\rho}$ is the second fundamental tensor as defined [17] by

$$
K_{\mu \nu}^{\rho}=\eta_{\nu}^{\sigma} \bar{\nabla}_{\mu} \eta_{\sigma}^{\rho}
$$

For the NG string $T^{\mu \nu}$ is simply proportional to the first fundamental tensor $\eta^{\mu \nu}$, and the extrinsic evolution equation (9) by itself constitutes the complete set of dynamical equations. However, in the elastic case, the surface stress energy tensor is slightly more complicated;

$$
T^{\mu \nu}=L \eta^{\mu \nu}+\mathcal{K} c^{\mu} c^{\nu}
$$

involving a surface current $c^{\mu}$ given by

$$
c^{\mu}=\frac{\psi}{\mathcal{K}} \bar{\nabla}^{\mu} \varphi=\frac{\psi}{\mathcal{K}} x_{, i}^{\mu} \gamma^{i j} \varphi, j
$$

Now the full set of dynamical equations is given by the extrinsic evolution equation (8), together with the surface current conservation law

$$
\bar{\nabla}_{\mu} c^{\mu}=0
$$


In the chiral case these equations also apply, though they must be supplemented by the constraint $w=0$, which means that the current $c^{\mu}$ is null. In this null current limit, the Lagrangian $L$ tends to a fixed value specified by the relevant Kibble mass scale $m$ say, and for the standard choice of $\kappa_{0}$ the quantity $\mathcal{K}$ tends to unity according to the specifications

$$
L\{0\}=-m^{2}, \quad \mathcal{K}\{0\}=1
$$

(see section 2a). Thus in the chiral case the stress energy tensor will take the form

$$
T^{\mu \nu}=-m^{2} \eta^{\mu \nu}+c^{\mu} c^{\nu}
$$

subject to the nullity condition

$$
c^{\mu} c_{\mu}=0,
$$

where the current is given simply by

$$
c^{\mu}=\psi \bar{\nabla}^{\mu} \varphi=\psi x_{, i}^{\mu} \gamma^{i j} \varphi_{, j}
$$

Recall that whereas $\psi$ is held constant in the analogous formula (11) for the elastic case, it may vary in (16) for the chiral case. However, the on shell variations of $\psi$ are severely restricted since on a two dimensional world sheet, the nullity condition (15) (i.e. $w=0$ ), automatically imposes that the phase field must satisfy the harmonicity condition

$$
\left(\|\gamma\|^{1 / 2} \gamma^{i j} \varphi_{, i}\right)_{, j}=0
$$

It can thus be seen from the internal coordinate version of the current conservation law (12), namely

$$
\left(\|\gamma\|^{1 / 2} \gamma^{i j} \psi \varphi, i\right), j=0,
$$

that the auxiliary field $\psi$ must satisfy the dynamical evolution condition

$$
\gamma^{i j} \varphi_{, i} \psi_{, j}=0
$$

which implies that $\psi$ will be restricted on shell to be a function just of $\varphi$.

\section{2a. Further properties of chiral strings and different actions}

Whereas different equations of state for $L\{w\}$ give qualitatively different elastic string models, the chiral model on the other hand is unique (just as in the NG case), modulo the choice of the fixed mass scale $m$. Thus there is no loss of generality in taking the chiral string equation of state to have the simple linear form originally proposed by Witten [18, namely

$$
L=-m^{2}-\frac{1}{2} w \Rightarrow \mathcal{K}=1
$$

However, it is instructive to consider the many different allowed alternatives which may, for instance, make the integrability property of chiral string mentioned above

more transparent. Indeed one can see that the equations of motion (15) and (18) as 
well as the stress energy tensor (14) for the chiral string can be obtained from any action of the form

$$
L=-m^{2}-\frac{1}{2} w+a_{2} w^{2}+a_{3} w^{3}+\ldots,
$$

where the $a_{n}(n=2,3, \ldots)$ are arbitrary numerical constants. These additional terms do not contribute on shell since in the equations of motion they lead to terms proportional to $w^{n-1}$ which vanish in the chiral limit $w=0$. Off-shell, however, they are important. As we now explain, a particularly useful version of this action for many reasons is the non-linear form

$$
L=-m^{2}\left(1+w / m^{2}\right)^{1 / 2} \Rightarrow \mathcal{K}=\left(1+w / m^{2}\right)^{1 / 2}
$$

which we shall often call the 'square root' action.

First consider action (22) for arbitrary (generally non-zero) $w$. From the corresponding stress-energy tensor it follows that the energy density $U$ and tension $T$ satisfy

$$
U T=\text { const. }
$$

Hence the two sound speeds $c_{E}$ and $c_{L}$ (respectively the transverse "wiggle" and longitudinal "woggle" sound speeds [29]) defined by

$$
c_{L}^{2}=\frac{T}{U} \quad, \quad c_{E}^{2}=-\frac{d T}{d U}
$$

are equal for action (22). (For a NG string, $c_{E}=1$ whilst $c_{L}$ has no analogue.) This special case $c_{L}=c_{E}$ characterises the transonic elastic string model that was already known to be integrable for any $w$ [30. Thus, turning now to the chiral case, once one has realised the equivalence of actions (20) and (22) it comes as no surprise that the chiral action is also integrable (as was first proved in reference 25] though on the basis of the action in the form (201) ).

A second feature of the action corresponding to the Lagrangian given in (22) is that it can viewed as a Kaluza-Klein projection of a NG action in a space-time of one higher dimension [31, 32]. Indeed

$$
\mathcal{I}=-m^{2} \int \mathrm{d}^{2} \sigma\|\gamma\|^{1 / 2}\left(1+w / m^{2}\right)^{1 / 2}=-m^{2} \int \mathrm{d}^{2} \sigma\|\Gamma\|^{1 / 2}
$$

where $\Gamma_{i j}$ is now the projection of the $(d+2)$-dimensional metric

$$
G_{\mathrm{AB}}=\left(\begin{array}{cc}
g_{\mu \nu} & 0 \\
0 & \psi^{2} / m^{2}
\end{array}\right)
$$

with the brane embedding is given by

$$
q^{\mathrm{A}}=\left(\begin{array}{c}
x^{\mu}\left(\sigma^{i}\right) \\
\varphi\left(\sigma^{i}\right)
\end{array}\right),
$$

so that $\Gamma_{i j}=\partial_{i} q^{\mathrm{A}} \partial_{j} q^{\mathrm{B}} G_{\mathrm{AB}}$. 
Finally it is interesting to observe that the action coming from (22) can be put into a Polyakov-like form - that is, a form which depends linearly on $\gamma_{i j}$ — but this is not possible for the action coming from (20): in the appendix we demonstrate this using Dirac's Hamiltonian formalism for contrained systems [33, 34 and also discuss the algebra of constraints. As expected, the result for the Polyakov action is

$$
\begin{aligned}
\mathcal{I} & =-m^{2} \int \mathrm{d}^{2} \sigma\|\gamma\|^{1 / 2}\left(1+w / m^{2}\right)^{1 / 2} \\
& =-\frac{m^{2}}{2} \int \mathrm{d}^{2} \sigma\|h\|^{1 / 2} h^{i j}\left(g_{\mu \nu} \partial_{i} x^{\mu} \partial_{j} x^{\nu}+\frac{\psi^{2}}{m^{2}} \partial_{i} \varphi \partial_{j} \varphi\right)
\end{aligned}
$$

where $h^{i j}$ is now an auxilliary metric field (again see the appendix). Note that this Polyakov action is reparametrisation and scale invariant so that coordinates can be chosen such that $h_{i j}=\eta_{i j}$ where $\eta_{i j}$ is the Minkowski metric. As for the Nambu string [35], this could be a starting point to discuss a possible quantization of the chiral string [36].

\section{General canonical symplectic structure}

Our aim is to subject the elastic and chiral string models to a canonical analysis of the kind described in the preceding article [1]. However, before doing so, we summarise in this section the main features of the canonical analysis. The following discussion is general and is not restricted to a particular string model.

Consider the general case of a worldsheet action density

$$
\mathcal{L}=\|\eta\|^{1 / 2} L
$$

in which the Lagrangian density $L$ depends on a set of field components $q^{\mathrm{A}}$ and of their surface deriatives $q_{, i}^{\mathrm{A}}=\partial_{i} q^{\mathrm{A}}=\partial q^{\mathrm{A}} / \partial \sigma^{i}$ where the $\sigma^{i}$ are the worldsheet coordinates. In this discussion we do not restrict the worldsheet dimension to 2 , so that the formalism presented here will be applicable not just to strings but also to higher dimensional branes. The field variables $q^{\mathrm{A}}$ can be of internal or external kind, the most obvious example of the latter kind being the background coordinates $x^{\mu}$ themselves. (In the case of the elastic string $q^{\mathrm{A}}=\left(x^{\mu}, \varphi\right)$.)

Subject to the understanding that the internal coordinates are held fixed,

$$
\delta \sigma^{i}=0
$$

the generic action variation,

$$
\delta \mathcal{L}=\mathcal{L}_{\mathrm{A}} \delta q^{\mathrm{A}}+p_{\mathrm{A}}^{i} \delta q_{, i}^{\mathrm{A}},
$$

specifies a set of partial derivative components $\mathcal{L}_{\mathrm{A}}$ and an associated set of generalised momentum components $p_{\mathrm{A}}^{i}$, which can be used to construct a corresponding pseudoHamiltonian scalar density

$$
\tilde{\mathcal{H}}=p_{\mathrm{A}}^{i} q_{, i}^{\mathrm{A}}-\mathcal{L} .
$$

(The covariance of such a pseudo-Hamiltonian distinguishes it from the ordinary kind of Hamiltonian, which depends on the introduction of some preferred time foliation as for instance the appendix of this article, equation (744).) 
According to the variational principle, the dynamically admissible "on shell" configurations are those characterised by the vanishing of the Eulerian derivative given by

$$
\frac{\delta \mathcal{L}}{\delta q^{\mathrm{A}}}=\mathcal{L}_{\mathrm{A}}-p_{\mathrm{A}, i}^{i}
$$

For an on-shell configuration, i.e. when the dynamical equations

$$
\frac{\delta \mathcal{L}}{\delta q^{\mathrm{A}}}=0
$$

are satisfied, the pseudo-Hamiltonian variation will take the form

$$
\delta \tilde{\mathcal{H}}=q_{, i}^{\mathrm{A}} \delta p_{\mathrm{A}}^{i}-p_{\mathrm{A}, i}^{i} \delta q^{\mathrm{A}} .
$$

Thus the Lagrangian variation can be written as a pure surface divergence

$$
\delta \mathcal{L}=\vartheta^{i}{ }_{, i}
$$

where $\vartheta^{i}$ is the generalised Liouville 1-form (on the configuration space cotangent bundle) defined by

$$
\vartheta^{i}=p_{\mathrm{A}}^{i} \delta q^{\mathrm{A}}
$$

Equation (37) shows that the Liouville 1-form is interpretable as a surface current that will be conserved (in the sense of having vanishing surface divergence) provided it is constructed from a perturbation that generates a local symmetry of the Lagrangian density, i.e. such that $\delta \mathcal{L}=0$. In the general case it is not conserved.

We can go on to construct a surface current that will always be conserved when the relevant dynamical equations are satisfied. This is done by taking the exterior differential of the Liouville form, i.e. by evaluating the commutator of a pair of successive independent variations, in the manner described in detail in the preceding article [1]. This exterior variation procedure provides us with the closed (since manifestly exact) symplectic 2-form expressible as

$$
\varpi^{i}=\delta \wedge \vartheta^{i}=\delta p_{\mathrm{A}}^{i} \wedge \delta q^{\mathrm{A}}
$$

where we have used the wedge symbol $\wedge$ to indicate antisymmetrisation with respect to the two independent variations involved. (Many authors prefer to use an extreme kind of abbreviation scheme in which the wedge symbol is omitted, but - as discussed in [1] - the use of such ultra-concise notation can lead to confusion in cases involving symmetrisation as well as antisymmetrisation). It is easy to verify that whenever both perturbations satisfy the relevant perturbed field equations

$$
\delta\left(\frac{\delta \mathcal{L}}{\delta q^{\mathrm{A}}}\right)=0,
$$

the symplectic 2 -form will be interpretable as a conserved worldsheet current in the sense that it will satisfy

$$
\varpi_{, i}^{i}=0
$$


We now follow the strategy of [11] and (as far as possible) work with quantities that are purely tensorial with respect to the background space. Hence we translate the surface current densities, whose components $\vartheta^{i}$ and $\varpi^{i}$ depend on the choice of the internal coordinates $\sigma^{i}$, into corresponding quantities which have stricly vectorial background coordinate components. These are given by

$$
\Theta^{\nu}=\|\gamma\|^{-1 / 2} x_{, i}^{\nu} \vartheta^{i} \quad \Omega^{\nu}=\|\gamma\|^{-1 / 2} x_{, i}^{\nu} \varpi^{i}
$$

The divergence law (37) is now rewritten in terms of the vectorial version of the Liouville form as

$$
\bar{\nabla}_{\nu} \Theta^{\nu}=\|\eta\|^{-1 / 2} \delta\left(L\|\gamma\|^{1 / 2}\right) .
$$

Similarly the conservation law (40) simply becomes

$$
\bar{\nabla}_{\nu} \Omega^{\nu}=0
$$

\section{Comoving reference system for the simply elastic case.}

We now apply the previous formalism to the elastic string for which $q^{\mathrm{A}}=\left(x^{\mu}, \varphi\right)$, and $p^{\mathrm{A}}=\left(p^{\mu}, \pi\right)$ where $p_{\mu}=\delta \mathcal{L} / \delta \dot{x}^{\mu}, \pi=\delta \mathcal{L} / \delta \dot{\varphi}$.

The meaning of the convention (31) that the local variation $\delta$ should be evaluated at a fixed value of the internal coordinates $\sigma^{i}$ depends on how these coordinates are chosen. For explicit solutions of the field equations it may be most convenient to choose cordinates that are constant along characteristics - as discussed in section 2a, this is particularly particularly true in the transonic case for which the extrinsic and intrinsic characteristics coincide. However, in the generic elastic case, the most convenient option is to take the internal coordinates to be comoving with respect to the intrinsic material structure. For a generic $p$-brane this is specified in terms of a set of $p$ independent scalar fields; for an ordinary perfect fluid or more general elastic solid in 4-dimensional spacetime one has $p=3$; whilst in the elastic string case we are concerned with here one simply has $p=1$ with the field in question being the phase scalar $\varphi$. Assuming the absence of singularities where the phase gradient is not just null but actually vanishes, there will be no loss of generality in postulating that the internal coordinates are comoving with respect to $\varphi$. In other words, we postulate that there is a vanishing variation, $\delta \varphi=0$, and hence also vanishing gradient variation, $\delta \varphi_{, i}=0$ with respect to these coordinates.

Subject to the choice of such a comoving internal reference system, the only remaining independent field variables in the elastic string model are the background coordinates $x^{\mu}$, so the generic variation (32) will take the specific form

$$
\delta \mathcal{L}=\mathcal{L}_{\mu} \xi^{\mu}+p_{\mu}^{i} \xi_{, i}^{\mu}
$$

using the notation

$$
\xi^{\mu}=\delta x^{\mu}
$$

for the relevant displacement vector. The corresponding expression for the Liouville form (38) is given by

$$
\vartheta^{i}=p_{\mu}^{i} \xi^{\mu}
$$


while for the symplectic 2-form (39) is given by the expression

$$
\varpi^{i}=\delta_{\Gamma} p_{\mu}^{i} \wedge \xi^{\mu}
$$

Here the parallel variation ${ }_{\Gamma} p_{\mu}{ }^{i}$ is given in terms of the simple momentum variation $\delta p_{\mu}^{i}$ by

$$
{ }_{\Gamma}^{\delta} p_{\mu}^{i}=\delta p_{\mu}^{i}-\Gamma_{\mu}^{\nu} \rho p_{\nu}^{i} \xi^{\rho},
$$

where $\Gamma_{\mu}{ }^{\nu}$ are the Riemannian connection components. In view of the symmetry of the latter it actually makes no difference whether $\delta_{\Gamma} p_{\mu}{ }^{i}$ or $\delta p_{\mu}{ }^{i}$ is used in (49), but use of the parallel variation is more convenient for our next step, which is the evaluation of the corresponding background tensorial formulae.

Since the background coordinate displacement will effect the Lagrangian only via the change of the induced metric, the resulting variation will be given simply by

$$
\delta \mathcal{L}=\frac{1}{2}\|\eta\|^{1 / 2} T_{\mathrm{L}}^{\mu \nu} \delta g_{\mu \nu}
$$

where $T^{\mu \nu}$ is the surface stress energy tensor defined in (17), and $\delta g_{\mu \nu}$ is the Lagrangian variation of the metric, meaning the change with respect to a coordinate system that is comoving with respect to the displacement. In the absence of any Eulerian variation (meaning that the spacetime background is held fixed) the Lagrangian variation is just given by the corresponding Lie derivative:

$$
{ }_{\mathrm{L}} g_{\mu \nu}=\vec{\xi} \mathcal{E} g_{\mu \nu}=2 \nabla_{(\mu} \xi_{\nu)} .
$$

Comparing (50) with the canonical variation formula (45) the partial derivatives involved can be read out as

$$
\mathcal{L}_{\mu}=\|\gamma\|^{1 / 2} \Gamma_{\mu \rho}^{\nu} T_{\nu}{ }^{\rho}
$$

and

$$
p_{\mu}^{i}=\|\gamma\|^{1 / 2} T_{\mu \nu} \eta^{i j} x_{, j}^{\nu} .
$$

It is thus immediately apparent that the pseudo-Hamiltonian density (33) will be given by

$$
\|\gamma\|^{-1 / 2} \tilde{\mathcal{H}}=T_{\nu}{ }^{\nu}-L
$$

and that the vectorial version (42) of the Liouville current will be given simply by

$$
\Theta^{\nu}=T_{\mu}^{\nu} \xi^{\mu}
$$

\section{Evaluation of the symplectic current.}

In order to evaluate the symplectic current a little more work is required. To start with (as in the preceeding work [1]) it is convenient to go over from parallel variations to the corresponding Lagrangian variations using the relations

$$
\underset{\Gamma}{\delta} x_{, i}^{\mu}=x_{, i}^{\nu} \nabla_{\nu} \xi^{\mu}
$$


and

$$
{ }_{\Gamma}^{\delta} T_{\mu}{ }^{\nu}=\underset{\mathrm{L}}{\delta} T_{\mu}{ }^{\nu}+T_{\rho}^{\nu} \nabla_{\mu} \xi^{\rho}-T_{\mu}{ }^{\rho} \nabla_{\rho} \xi^{\nu} .
$$

The vectorial version (43) of the symplectic current is thereby obtained in the form

$$
\Omega^{\nu}=\|\gamma\|^{-1 / 2} x_{, i}^{\nu}{ }_{\Gamma} p_{\mu}^{i} \wedge \xi^{\mu}
$$

with

$$
\|\gamma\|^{-1 / 2} x_{, i}^{\nu}{ }_{\Gamma} p_{\mu}^{i}={ }_{\mathrm{L}} T_{\mu}{ }^{\nu}-T_{\rho}^{\nu} \nabla_{\mu} \xi^{\rho}+T_{\mu}{ }^{\nu} \bar{\nabla}_{\rho} \xi^{\rho} .
$$

The advantage of Lagrangian variations is their convenience for relating the relevant intrinsic physical quantities via the appropriate equations of state. Following the example of Friedman and Schutz [37] in the context of ordinary relativistic fluid dynamics, we use the second order derivative of the action with respect to the background metric to obtain the hyper Cauchy tensor (generalised elasticity tensor) according to the prescription

$$
C^{\mu \nu \rho \sigma}=\|\gamma\|^{-1 / 2} \frac{\partial\left(T^{\mu \nu}\|\gamma\|^{1 / 2}\right)}{\partial g_{\rho \sigma}}=C^{\rho \sigma \mu \nu}
$$

The Lagrangian variation of the surface stress energy tensor will thus be obtained in the form

$$
{ }_{\mathrm{L}}^{\delta} T^{\mu \nu}=\left(C^{\mu \nu \rho \sigma}-\frac{1}{2} T^{\mu \nu} \eta^{\rho \sigma}\right) \underset{\mathrm{L}}{\delta} g_{\rho \sigma} .
$$

The symplectic current can thereby be expressed in purely tensorial form as

$$
\Omega^{\nu}=\left(2 C_{\mu \rho}^{\nu \sigma} \bar{\nabla}_{\sigma} \xi^{\rho}+T^{\nu \rho} \bar{\nabla}_{\rho} \xi_{\mu}\right) \wedge \xi^{\mu}
$$

Using the explicit expression (10) for the surface stress energy tensor in a generic elastic string model, the corresponding expression for the required hyper Cauchy tensor is obtainable from the definition (60) in the form

$$
\begin{aligned}
C^{\mu \nu \rho \sigma}=L\left(\frac{1}{2} \eta^{\mu \nu} \eta^{\rho \sigma}-\eta^{\mu(\rho} \eta^{\sigma) \nu}\right)+\frac{\mathcal{K}}{2}\left(\eta^{\mu \nu} c^{\rho} c^{\sigma}+\eta^{\rho \sigma} c^{\mu} c^{\sigma}-4 c^{(\mu} \eta^{\nu)(\rho} c^{\sigma)}\right) \\
+\mathcal{K}^{2} \frac{\mathrm{d} \mathcal{K}}{\mathrm{d} w} c^{\mu} c^{\nu} c^{\rho} c^{\sigma} .
\end{aligned}
$$

\section{The chiral case}

As remarked above, the only way in which the off shell action for the chiral model differs from that of the generic elastic case is that instead of being held constant the auxiliary field $\psi$ is treated as a free variable. However, as its gradient is not involved in the action, this extra variable will not give rise to any corresponding momentum contribution, so the formulae of the two preceding sections will remain valid for the chiral model as characterised on shell by the current nullity condition (15) and the coresponding restrictions (13).

It was remarked that for a given value of the overall normalisation as fixed by the mass scale $m$, the same unique chiral model with the same on shell stress energy 
tensor (14) is obtained independently of the equation of state. Notice, however, that due to the presence of the final term proportional to $\mathrm{d} \mathcal{K} / \mathrm{d} w$, the formula (63) for the hyper Cauchy tensor gives a result that does depend on the choice of equation of state even in the chiral limit for which $w=0$ and $\mathcal{K}=1$. The simplest possibility is provided by the choice [25] of Witten's simple linear equation of state (20), which gives $\mathrm{d} \mathcal{K} / \mathrm{d} w=0$, so that the final term in (63) will drop out altogether. However, for other choices such as the more useful one (22) discussed in section 2, there will be an extra term proportional to $c^{\mu} c^{\nu} c^{\rho} c^{\sigma}$ with an arbitrary proportionality constant. Although it is uniquely defined on shell, the reason why the chiral model does not provide a unique specification of the on shell hyper Cauchy tensor is that the range of variation in the relevant partial derivative formula (61) is restricted by the requirement that, in order to preserve the nullity condition (15), the allowable displacements must be such as to ensure that the Lagrangian metric variation satisfies the on shell chiral variation condition

$$
c^{\rho} c^{\sigma}{ }_{\mathrm{L}} g_{\rho \sigma}=0
$$

However, for this same reason, the final term in (63) will provide no contribution to the on shell value of the corresponding symplectic tensor, whose value for the chiral model will thus be given unambiguously by substitution in (62) of the expression obtained from the linear action (20), namely

$$
C^{\mu \nu \rho \sigma}=m^{2}\left(\eta^{\mu(\rho} \eta^{\sigma) \nu}-\frac{1}{2} \eta^{\mu \nu} \eta^{\rho \sigma}\right)+\frac{1}{2}\left(\eta^{\mu \nu} c^{\rho} c^{\sigma}+\eta^{\rho \sigma} c^{\mu} c^{\sigma}-4 c^{(\mu} \eta^{\nu)(\rho} c^{\sigma)}\right) .
$$

In terms of the orthogonal projector $\perp_{\nu}^{\mu}=g^{\mu}{ }_{\nu}-\eta^{\mu}{ }_{\nu}$, it can be seen that this leads to an expression giving the conserved symplectic current for the chiral string model in the form

$$
\Omega^{\nu}=\left(c_{\mu} c_{\rho} \eta^{\nu \sigma}+2 T_{\mu}^{[\sigma} \eta_{\rho}^{\nu]}-T^{\nu \sigma} \perp_{\mu \rho}\right) \xi^{\mu} \wedge \bar{\nabla}_{\sigma} \xi^{\rho},
$$

in which the chiral stress energy tensor is given by (14).

\section{Conclusion}

Our aim in this paper was first and foremost to calculate the symplectic current central to the covariant canonical analysis of Witten and others [2, 3, 4, 5, 6, 7, 8] for generic elastic and chiral string models. The purpose of this analysis was as a precursor to a covariant quantization.

Our work is based on the concise and efficient covariant analysis developed in [11. 12, 13, rather than the more cumbersome frame dependent methods which have been used by others in the case of the simpler NG string. Our results are presented in sections 5 and 6 for the elastic and chiral strings respectively. In the process, in section $2 \mathrm{a}$ and the appendix, we also studied with care the different formulations of the chiral string model. We showed the equivalence of the Witten action (used in most studies to date), with the square root action (equation (22) ) and in this way we were able to understand in a different way the integrability properties of the chiral string. 


\section{Acknowledgements}

D.Steer would like to thank M. Ruiz-Altaba for many discussions on the quantisation of chiral strings and the appendix of this article.

\section{A. Appendix}

In this appendix we demonstrate how the Polyakov-action (29) can be obtained from the original square root action (22) using Dirac's Hamiltonian formalism for contrained systems [33, 34]. The bulk of this appendix is valid for arbitrary $w$ and hence for general elastic string models: the special chiral limit $w=0$ will be discussed after equation (82) where the Poisson algebra of constraints - including the chiral one - is studied. (For a different work on constrained superconducting membranes, see [38.)

Our starting point is the action (22)

$$
\mathcal{I}=\int \mathrm{d}^{2} \sigma \mathcal{L}=-m^{2} \int \mathrm{d}^{2} \sigma\|\gamma\|^{1 / 2}\left(1+w / m^{2}\right)^{1 / 2}
$$

in an arbitrary background metric $g_{\mu \nu}$ and brane embedding $x^{\mu}$. Let the two worldsheet coordinates be denoted by $\sigma^{(0,1)} \equiv(\tau, \sigma)$ with $\tau$ time-like, and ${ }^{\prime}=\partial_{1}$ and ${ }^{*}=\partial_{0}$. The canonical momentum densities $p_{\mu}=\delta \mathcal{L} / \delta \dot{x}^{\mu}, \pi=\delta \mathcal{L} / \delta \dot{\varphi}$ and $p_{\psi}=\delta \mathcal{L} / \delta \dot{\psi}=0$ are given by

$$
\begin{aligned}
p_{\mu} & =\frac{m^{4}}{\mathcal{L}}\left[\left(\dot{x} \cdot x^{\prime}\right) x_{\mu}^{\prime}-x^{\prime 2} \dot{x}_{\mu}+\frac{\psi^{2}}{m^{2}}\left(x_{\mu} \dot{\varphi} \varphi^{\prime}-\dot{x}_{\mu} \varphi^{\prime 2}\right)\right] \\
\pi & =-\psi^{2} \frac{m^{2}}{\mathcal{L}}\left[x^{\prime 2} \dot{\varphi}-\left(\dot{x} \cdot x^{\prime}\right) \varphi^{\prime}\right]
\end{aligned}
$$

and the phase space of the system is $\left\{x^{\mu}, \varphi, p_{\mu}, \pi\right\}$. It follows directly from these definitions of $p_{\mu}$ and $\pi$ that there two are primary constraints independent of $\dot{x}$ and $\dot{\varphi}$. One (see equation (73) below) is straightforward to deduce. In order to find the other, it is easiest express both $p^{2}$ and $\pi^{2}$ in terms of $w=\psi^{2} \gamma^{i j} \varphi_{, i} \varphi_{, j}$ defined in (2), leading to

$$
\begin{aligned}
-\frac{w}{m^{6}}\left[p^{2}+\psi^{2} \varphi^{\prime 2} m^{2}\right] & =x^{\prime 2}+\frac{1}{m^{4}}\left[p^{2}+2 \psi^{2} \varphi^{\prime 2} m^{2}\right] \\
w\left[x^{\prime 2} \psi^{2}+\frac{\pi^{2}}{m^{2}}\right] & =\psi^{4} \varphi^{\prime 2}-\pi^{2}
\end{aligned}
$$

Eliminating $w$ between these equations gives the other primary constraint, so that the two constraints are

$$
\begin{aligned}
& \mathcal{H}_{1} \equiv p^{2}+\frac{m^{2} \pi^{2}}{\psi^{2}}+m^{4} x^{\prime 2}+m^{2} \psi^{2} \varphi^{\prime 2}=0, \\
& \mathcal{H}_{2} \equiv p_{\mu} x^{\prime \mu}+\pi \varphi^{\prime}=0 .
\end{aligned}
$$

The Nambu-Goto limit is obtained when $\psi \rightarrow 0$ (note that $\pi^{2} \sim \psi^{4}$ ) in which case these expressions reduce to the standard constraints [34 for the bosonic string. 
Had we started instead from the linear Witten action (20) and calculated the new momenta, it would have followed that the second constraint (73) still holds. On the other hand, a constraint of the first form linking $p^{2}$ and $\pi^{2}$ can no longer be obtained: though one can still express $p^{2}$ as a function of $w$ in a manner analogous to (70), there is no analogue of (71) for the linear Witten action. Indeed $\pi^{2}$ is now depends on $\dot{\varphi}$ and $\dot{x}^{\mu}$ in a combination that can no longer be expressed solely in terms of $w$.

Going back to the square root action, it follows from the above expressions that the canonical Hamiltonian density vanishes

$$
\mathcal{H}_{c} \equiv \pi \dot{\varphi}+\dot{x}_{\mu} p^{\mu}-\mathcal{L}=0
$$

As explained by Dirac [33, 34, this Hamiltonian is ambiguous since one is free to add arbitrary multiples of the vanishing primary constraints and secondary constraints. These secondary constraints arise from imposing that the primary constraints should be preserved under time evolution [33. Here, given that $\mathcal{H}_{c}=0$ and that the Poisson brackets of the $\mathcal{H}_{1,2}$ are closed (see equations (79)-(81) below), it follows that there are no secondary constraints. Hence the 'total' Hamiltonian density 33, 34] which determines the dynamics of the system is simply a linear combination of the primary constraints,

$$
\mathcal{H} \equiv \mathcal{H}_{c}+\frac{\lambda}{2 m^{2}} \mathcal{H}_{1}+\mu \mathcal{H}_{2}=\frac{\lambda}{2 m^{2}} \mathcal{H}_{1}+\mu \mathcal{H}_{2},
$$

where $\mu$ and $\lambda$ are dimensionless Lagrange multipliers. We now briefly turn away from the phase space approach to calculate the (Polyakov) Lagrangian

$$
\mathcal{L}_{P} \equiv \dot{x}_{\mu} p^{\mu}+\dot{\varphi} \pi-\mathcal{H}
$$

corresponding to (75). From the equation of motion $\dot{x}_{\mu}\left(\tau, \sigma^{\prime}\right)=\int d \sigma\left\{x_{\mu}\left(\tau, \sigma^{\prime}\right), \mathcal{H}(\tau, \sigma)\right\}$ (with a similar one for $\varphi$ ) it follows that $p_{\mu}=\left(m^{2} / \lambda\right)\left(\dot{x}_{\mu}-\mu x_{\mu}^{\prime}\right)$, and similarly $\pi=-\left(\psi^{2} / \lambda\right)\left(\mu \varphi^{\prime}-\dot{\varphi}\right)$. These enable all the momenta to be eliminated from $\mathcal{L}_{P}$ of equation (76), and one finds in the particular case of the square root action

$$
\mathcal{I}=\int \mathrm{d}^{2} \sigma \mathcal{L}_{P}=-\frac{m^{2}}{2} \int \mathrm{d}^{2} \sigma\|h\|^{1 / 2} h^{i j}\left(g_{\mu \nu} \partial_{i} x^{\mu} \partial_{j} x^{\nu}+\frac{\psi^{2}}{m^{2}} \partial_{i} \varphi \partial_{j} \varphi\right)
$$

where the components of $h_{i j}$ are simply expressible in terms of $\lambda$ and $\mu$ :

$$
\sqrt{-h} h^{00}=-\frac{1}{\lambda}, \quad \sqrt{-h} h^{01}=\frac{\mu}{\lambda}, \quad \sqrt{-h} h^{11}=\lambda-\frac{\mu^{2}}{\lambda} .
$$

This is the result given in (29). Note that action (77) has reparametrization and Weyl scale invariance generated by the two primary constraints $\mathcal{H}_{1,2}$, and reflected in the two free parameters $\lambda$ and $\mu$. For instance, an appropriate choice of coordinates can set $h_{i j}=\eta_{i j}$ where $\eta_{i j}$ is the Minkowski metric (or more simply one can choose $\lambda=1$ and $\mu=0$ in (178)). As for the Nambu string [35], this is one appropriate starting point with which to quantize the chiral string [36].

As noted above, this result is a special property of the square root action. For example, in the case of the linear (Witten) action, equation (20), only the primary 
constraint $\mathcal{H}_{2}$ exists so that the 'full' Hamiltonian would now contain a single term, $\mathcal{H}=\mu \mathcal{H}_{2}$. Following a similar procedure to that above shows that one would no longer be able to eliminate the momenta in order to calculate the corresponding Lagrangian. (The equations of motion do not give equations for $p_{\mu}$ and $\pi$ but rather the equalities $\dot{x}_{\nu}=\mu x_{\nu}^{\prime}$ and $\dot{\varphi}=\mu \varphi^{\prime}$.)

We now make a comment about the algebra of constraints for the square root action. As for the Nambu string 34] (and also as previously noted), it follows from the equations of motion that generators $\mathcal{H}_{1}$ and $\mathcal{H}_{2}$ form a closed algebra (i.e. they are first class constraints):

$$
\begin{aligned}
& \left\{\mathcal{H}_{1}(\sigma), \mathcal{H}_{1}\left(\sigma^{\prime}\right)\right\} \propto \mathcal{H}_{2} \frac{\partial}{\partial \sigma} \delta\left(\sigma-\sigma^{\prime}\right), \\
& \left\{\mathcal{H}_{1}(\sigma), \mathcal{H}_{2}\left(\sigma^{\prime}\right)\right\} \propto \mathcal{H}_{1} \frac{\partial}{\partial \sigma} \delta\left(\sigma-\sigma^{\prime}\right), \\
& \left\{\mathcal{H}_{2}(\sigma), \mathcal{H}_{2}\left(\sigma^{\prime}\right)\right\} \propto \mathcal{H}_{2} \frac{\partial}{\partial \sigma} \delta\left(\sigma-\sigma^{\prime}\right) .
\end{aligned}
$$

Is it also consistent to impose the chiral constraint at the algebraic level? As noted in section 2 , the chiral constraint $\partial \mathcal{L} / \partial \psi=0$ imposes the nullity condition

$$
w=0
$$

which we would like to express on the same footing as the primary constraints (72) and (173) - that is, in terms of momenta only. Let this constraint be denoted by $\mathcal{H}_{3}$. From equations (70) and (71), if therefore follows that

$$
\mathcal{H}_{3} \equiv \alpha\left[m^{4} x^{\prime 2}+p^{2}+2 \psi^{2} \varphi^{\prime 2} m^{2}\right]+\psi^{2} m^{2} \beta\left[\varphi^{\prime 2}-\frac{\pi^{2}}{\psi^{4}}\right]=0,
$$

for initially arbitrary $\alpha$ and $\beta$. Recall, however (section 2), that the null constraint $w=0$ is consistent with the dynamical equations, being conserved under time evolution. At the level of constraints, we demand that the chiral constraint $\mathcal{H}_{3}$ should also be conserved under time evolution - in other words, that it does not lead to a secondary constraint. Equivalently this imposes that the Poisson brackets of $\mathcal{H}_{3}$ with the other two constraints $\mathcal{H}_{1}, \mathcal{H}_{2}$ should be closed. In turn one can show that this is true only for $\alpha=1 / 2$ and $\beta=-\alpha(1 \pm \sqrt{2})$ in which case

$$
\begin{aligned}
\left\{\mathcal{H}_{3}(\sigma), \mathcal{H}_{1}\left(\sigma^{\prime}\right)\right\} & \propto \mathcal{H}_{2} \frac{\partial}{\partial \sigma} \delta\left(\sigma-\sigma^{\prime}\right), \\
\left\{\mathcal{H}_{2}(\sigma), \mathcal{H}_{3}\left(\sigma^{\prime}\right)\right\} & \propto \mathcal{H}_{3} \frac{\partial}{\partial \sigma} \delta\left(\sigma-\sigma^{\prime}\right), \\
\left\{\mathcal{H}_{3}(\sigma), \mathcal{H}_{3}\left(\sigma^{\prime}\right)\right\} & \propto \mathcal{H}_{2} \frac{\partial}{\partial \sigma} \delta\left(\sigma-\sigma^{\prime}\right) .
\end{aligned}
$$




\section{References}

[1] B. Carter, "Symplectic structure in brane mechanics", hep-th/0302084

[2] E. Witten, "Interacting field theory of open superstrings", Nucl. Phys. B276 (1986) 291.

[3] C. Crncovic, E. Witten, "Covariant description of canonical formalism in geometric theories" in 300 years of Gravitation, ed. S.W. Hawking, W. Israel (Cambridge U.P., 1987) 676-684.

[4] G.J Zuckerman, in Mathematical elements of string theory, ed. S.T. Yau (World scientific, Singapore, 1987).

[5] K-S. Soh, "Covariant symplectic structure of two-dimensional dilaton gravity" Phys. Rev. D49 (1994) 1906-1911.

[6] R. Cartas-Fuentevilla, "Symplectic current for the field perturbatios in dilaton axion gravity coupledwith Abelian fields" Phys. Rev.D57 (1998) 3443-3448.

[7] Y. Nutku, "Covariant symplectic structure of the complex Monge-Ampere equation" Phys. Lett.A268( 2000) 293. hep-th/0004164

[8] C. Rovelli, "Covariant Hamiltonian formalism for field theory: symplectic structure and Hamilton-Jacobi structure on the space G." gr-qc/0207043

[9] R. Cartas-Fuentevilla, "Identically closed two-form for covariant phase space quantisation of Dirac - Nambu - Goto p-branes in a curved space-time", Phys. Lett. B536 (2002) 283 - 288. hep-th/0204133

[10] R. Cartas-Fuentevilla "Global symplectic potentials in the Witten covariant phase space for bosonc extendons" Phys.Lett. B536 (2002) 289-293. hep-th/0204135

[11] B. Carter, "Perturbation dynamics for membranes and strings governed by the Dirac - Goto - Nambu action in curved space", Phys. Rev. D48 (1993) 4835-4838.

[12] R.A. Battye, B. Carter, "Gravitational Perturbations of Relativistic Membranes and Strings" Phys. Lett. B357 (1995) 29-35. hep-ph/9508300

[13] R.A. Battye, B. Carter, "Second order Lagrangian and symplectic current for gravitationally perturbed Dirac-Goto-Nambu strings and branes" Class. Quant.Grav. 17 (2000) 3325-3334. hep-th/9811075

[14] B. Carter, "Electromagnetic self interaction in strings" Phys.Lett. B404 (1997) 246-252. hep-th/9704210

[15] B. Carter, R.A. Battye, "Non-divergence of gravitational self-interactions for Goto-Nambu strings" Phys. Lett. B430 (1998) 49-53. hep-th/9803012 
[16] B. Carter, R.A. Battye, J.P. Uzan, "Gradient formula for linearly self-interacting branes" Com. Math. Phys. (to appear) hep-th/0204042

[17] B. Carter, "Stability and characteristic propagation speeds in superconducting cosmic and other cosmic string models", Phys. Lett. B228 (1989) 466-470.

[18] E. Witten, "Superconducting strings" Nucl. Phys. B249 (1985) 557-592

[19] R.L. Davis, E.P.S. Shellard, "The physics of vortex superconductivity II: charge, angular momentum, and the vorton", Phys. Letters B209, 485.

[20] B. Carter, X. Martin, "Dynamic instability criterion for circular string loops", Ann. Phys. 227 (1993) 151-171.

[21] B. Carter, P. Peter, A. Gangui, "Avoidance of collapse by circular currentcarrying cosmic strings", Phys. Rev. D55 (1997) 4647-4662 hep-ph/9609401.

[22] C.J.A.P.Martins, E.P.S.Shellard, "Vorton Formation", Phys. Rev. D57 (1998) 7155-7176 [hep-ph/9804378

[23] S.C. Davis, A.C. Davis, M. Trodden, "N=1 Supersymmetric Cosmic Strings", Phys. Lett. B405 (1997) 257-264 hep-ph/9702360

[24] B. Carter, A.C. Davis, "Chiral Vortons and Cosmological Constraints on Particle Physics", Phys. Rev. D61 (2000) 123501 hep-ph/9910560

[25] B. Carter, P. Peter, "Dynamics and integrability property of the chiral string model", Phys. Lett. B466 (1999) 41-49 hep-th/9905025

[26] A.C. Davis, T.W.B. Kibble, M. Pickles, D.A. Steer "Dynamics and properties of chiral cosmic strings in Minkowski space", Phys. Rev. D62 (2000) 083516 astro-ph/0005514

[27] J.J. Blanco-Pillado, K.D.Olum, A.Vilenkin "Dynamics of superconducting strings with chiral currents", Phys. Rev. D63 (2001) 103513 astro-ph/0004410

[28] D.A. Steer "Self-intersections and gravitational properties of chiral cosmic strings in Minkowski space", Phys. Rev. D63 (2001) 083517 astro-ph/0011233

[29] B. Carter, "Transonic elastic model for wiggly Goto-Nambu string," Phys. Rev. Lett. 74 (1995) 3098.

[30] B. Carter "Integrable Equation of State for Noisy Cosmic String", Phys. Rev. D 41 (1990) 3869-3872.

[31] B. Carter, "Covariant mechanics of strings and membranes", in The formation and evolution of cosmic strings, Editors G.Gibbons, S.Hawking and T.Vachaspati, Cambridge University Press, Cambridge 1990, page 143. 
[32] R. Cordero and E. Rojas, "Chiral superconducting membranes," Int. J. Mod. Phys. A 17 (2002) 73.

[33] P.A.M. Dirac, "Lectures on Quantum Mechanics", Yeshiva University, New York, 1964.

[34] A. Hanson, T. Regge and C. Teitelboim, "Constrained Hamiltonian systems", Accademia nazionale dei Lincei, Roma, 1976.

[35] M. B. Green, J. H. Schwarz and E. Witten, "Superstring Theory. Vol. 1: Introduction," Cambridge University Press, Cambridge 1987.

[36] M.Ruiz-Altaba, "Quantized chiral string", private communication, 2002.

[37] J. Friedman, B.A. Schutz, "On the stability of relativistic systems" Astrop. J. 200 (1975) 204-220.

[38] R. Cordero and E. Rojas, Phys. Lett. B470 (1999) 45. arXiv:astro-ph/0009139. 